\title{
NON-LINEAR SIMILARITY REVISITED
}

\author{
IAN HAMBLETON AND ERIK K. PEDERSEN
}

Let $G$ be a finite group and $V, V^{\prime}$ finite dimensional real orthogonal representations of $G$. Then $V$ is said to be topologically similar to $V^{\prime}\left(V \sim_{t} V^{\prime}\right)$ if there exists a homeomorphism $h: V \rightarrow V^{\prime}$ which is $G$-equivariant. If $V, V^{\prime}$ are topologically similar, but not linearly isomorphic, then such a homeomorphism is called a nonlinear similarity.

The topological classification of $G$-representations was first studied by de Rham [18]. He proved that if a topological similarity $h: V \rightarrow V^{\prime}$ of orthogonal representations preserves the unit spheres and restricts to a diffeomorphism between $S(V)$ and $S\left(V^{\prime}\right)$, then $V$ and $V^{\prime}$ are linearly isomorphic. In 1973, Kuiper and Robbin [11] obtained positive results on the general problem and conjectured that topological equivalence implies linear equivalence for all finite groups $G$. However, in 1981 Cappell and Shaneson [1] constructed the first examples of non-linear similarities. The simplest occurs for $G=\mathbb{Z} / 8$, but they also constructed a large class of examples for cyclic groups of the form $G=\mathbb{Z} / 4 q$. Further results can be found in [4], [2], [3], and [13].

On the other hand, Hsiang and Pardon [10] and Madsen and Rothenberg [12] independently proved the conjecture for all odd-order groups. In addition, the main theorem of [10] ruled out some non-linear similarities for even-order groups $G$.

The purpose of this paper is to give new restrictions on the existence of non-linear similarities using techniques from bounded topology.

Theorem A. Let $V, V^{\prime}$ be real orthogonal $G$-representations, where $G$ is a finite cyclic group. Suppose that $\operatorname{Res}_{H} V \cong \operatorname{Res}_{H} V^{\prime}$ for each proper subgroup $H \subsetneq G$, and that $V^{H}=V^{G}$ when $[G: H]=2$. Then $V \sim_{t} V^{\prime}$ if and only if $V \cong V^{\prime}$.

This result gives information about topological similarities for non-cyclic groups as well, since linear equivalence of representations is detected by character values. The formulation is also well-adapted to inductive arguments, and we get a new proof for the results of [10], [12].

Corollary B. Let $G$ be a finite group and $V, V^{\prime}$ be real orthogonal $G$-representations. Suppose that for each cyclic subgroup $C \subseteq G$ of 2-power order, the elements of $C$ act

The first author was partially supported by NSERC grant A4000

The second author was partially supported by NSF DMS 9104026 
either trivially on $W$, or freely away from $0 \in W$, for every irreducible $C$-submodule $W \subset \operatorname{Res}_{C} V$. Then $V \sim_{t} V^{\prime}$ if and only if $V \cong V^{\prime}$.

All previously contructed topological similarities of cyclic groups $G$ contain the non-trivial 1-dimensional representation (i.e. the representation with isotropy group $H \subset G$ of index 2), or are induced from such examples.

For example, suppose that $G=\mathbb{Z} / 4 q, q=2^{r-2}, r \geq 4$. Now let $V_{1}=t^{i}+t^{j}$, with $i \equiv 1 \bmod 4$ and $j \equiv \pm i \bmod 8$, and $V_{2}=t^{i+2 q}+t^{j+2 q}$ where $t$ denotes a faithful 2-dimensional representation of $G$. Let $\epsilon$ (resp. $\delta$ ) denote the 1-dimensional trivial (resp. non-trivial) representation of $G$. Then $t^{i}+t^{j}+\delta+\epsilon \sim_{t} t^{i+2 q}+t^{j+2 q}+\delta+\epsilon$ by [4, Thm. 1, Cor. (iii)]. But $\operatorname{Res}_{H} V_{1} \cong \operatorname{Res}_{H} V_{2}$ for every proper subgroup $H \subsetneq G$, so our result says that $V_{1} \oplus W$ is not topologically similar to $V_{2} \oplus W$ unless $\delta$ is a summand of $W$. More generally,

Corollary C. Suppose that $V_{1} \oplus W \sim_{t} V_{2} \oplus W$ is a non-linear similarity for a cyclic group $G$. Then for some subgroup $H \subseteq G, \operatorname{Res}_{H}\left(V_{1} \oplus W\right) \sim_{t} \operatorname{Res}_{H}\left(V_{2} \oplus W\right)$ is also a non-linear similarity and the non-trivial 1-dimensional $H$-representation is a summand of $\operatorname{Res}_{H} W$.

Our techniques also give information about the existence and classification of nonlinear similarities. We recently noticed that $[4$, Thm $1(\mathrm{i})]$ is incorrect as stated. For example for $G=\mathbb{Z} / 12$, there are no 6 -dimensional non-linear similarities. The problem is that the natural epimorphism $\pi: \mathbb{Z} / 4 q \rightarrow \mathbb{Z} / 2^{r}$ where $q=2^{r-2} s, s$ odd, does not induce an isomorphism $\pi_{*}: L^{p}\left(\mathbb{Z}[\mathbb{Z} / 4 q]^{-}\right) \rightarrow L^{p}\left(\mathbb{Z}\left[\mathbb{Z} / 2^{r}\right]^{-}\right)$as claimed in $[4$, p. 732 l. -8]. These topics will be discussed elsewhere [8].

Acknowledgement: Both authors would like to thank the Mittag-Leffler Institute for its hospitality in May, 1994 when this work was completed.

\section{PRELIMINARIES}

Suppose that $V \sim_{t} V^{\prime}$ with $\operatorname{Res}_{H} V \cong \operatorname{Res}_{H} V^{\prime}$ for each proper subgroup $H \subsetneq G$, and that $V^{H}=V^{G}$ whenever $[G: H]=2$. The result for free representations was proved in $[11]$ or $[1,8.1]$, and we assume by induction that Theorem $\mathrm{A}$ is proved for all groups of smaller order than $G$. Therefore, we may further assume

(1.1): that $V, V^{\prime}$ are topologically similar $G$-representations whose restrictions to every $H$-fixed set are linearly equivalent as $G / H$-representations, for each non-trivial subgroup $1 \neq H \subset G$

(1.2): that $V, V^{\prime}$ are neither free nor quotient $G$-representations, i.e. for each $g \in G$ there exists $v \in V$ such that $g v \neq v$, and there exists $0 \neq v \in V$ such that $g v=v$ for some $1 \neq g \in G$. 
Lemma 1.3. If $V \sim_{t} V^{\prime}$ satisfies the conditions above, then $V=V_{1} \oplus W$ and $V^{\prime}=$ $V_{2} \oplus W$, where $V_{1}, V_{2}$ are free $G$-representations and $W \neq\{0\}$ has no free summands.

Proof. For each subgroup $K \subseteq G$, let $V(K)$ denote the direct sum of all irreducible subrepresentations of $V$ with kernel $K$. The $G$-subspaces $V(K)$ are preserved by $G$-linear isomorphisms. We let $W$ be the direct sum of all the $V(K)$ for $K \neq 1$. From (1.2) it follows that $V^{H} \neq\{0\}$ for some subgroup $1 \neq H \subset G$ so $W \neq\{0\}$.

Write $V=V_{1} \oplus W$ where $V_{1}$ is a free representation. By assumption (1.1), $V^{H} \cong$ $\left(V^{\prime}\right)^{H}$ as $G$-representations for each $H \neq 1$. Then for the fixed sets we have

$$
V(K)^{H}=\left\{\begin{array}{ll}
V(K) & \text { if } H \subseteq K \\
0 & \text { otherwise. }
\end{array}\right. \text { and }
$$

This gives

$$
V^{H}=\bigoplus\{V(K): H \subseteq K\} \cong\left(V^{\prime}\right)^{H}=\bigoplus\left\{V^{\prime}(K): H \subseteq K\right\} .
$$

Therefore $V(K) \cong V^{\prime}(K)$ for each subgroup $K \neq 1$ and we can decompose $V^{\prime}=$ $V_{2} \oplus W$ with $V_{2}$ a free representation.

\section{Bounded Embedding Theorems}

For the reader's convenience we will include some material from [8]. First we will need a bounded version of results due to Browder and Wall on smoothing Poincaré embeddings in codimension $\geq 3$. Statements for compact smooth or PL manifolds are given in [20, 11.3], and the extension to topological manifolds is sketched on [20, p.245]. The published reference to Rourke and Sanderson's theorem, that the stabilization map

$$
F_{r} / \widetilde{\operatorname{Top}}_{r} \rightarrow F / T o p
$$

is a homotopy equivalence for $r \geq 3$ is $\left[19\right.$, Thm 2,4]. Here $F_{r}$ denotes the space of homotopy self-equivalences of the $(r-1)$-sphere, and $F=\lim _{\rightarrow} F_{r}$.

To state a bounded version, we need to define a finite bounded Poincaré embedding. Let $X$ be a metric space on which a finite group $G$ acts by (quasi)isometries. Let $Y \subset X$ be a closed $G$-invariant subspace, and let $M^{m} \rightarrow Y, V^{m+q} \rightarrow X$ be finite free bounded $G-C W$ Poincaré complexes [5, Def 2.7]. Then a finite bounded Poincaré embedding of $M$ in $V$ consists of (i) a $(q-1)$-spherical $G$-fibration $\xi$, with projection $p: E \rightarrow M$, (ii) a finite free bounded $G-C W$ Poincaré pair $(C, E) \rightarrow(X, Y)$, and (iii) a bounded $G$-homotopy equivalence $h: C \cup M(p) \rightarrow V$, bounded over $X$, where $M(p)$ is the mapping cylinder of $p$ and $C \cap M(p)=E$. Such a Poincaré embedding is "induced" by a locally flat topological embedding if the normal block bundle and complement to the embedding give data which are $G$-h-cobordant to those of the given Poincaré embedding. 
Theorem 2.1. Suppose given topological manifolds $M^{m}, V^{m+q}$ with free $G$-actions, and reference maps $M^{m} \rightarrow Y, V^{m+q} \rightarrow X$ giving finite free bounded $G-C W$ Poincaré complexes. If $m+q \geq 5$ and $q \geq 3$, then a finite bounded Poincaré embedding of $M$ in $V$ is induced by a locally flat topological $G$-embedding of $M \rightarrow V$.

Proof. The proof given in $[20, \S 11]$ generalizes using bounded surgery [6], and there is a relative version as given on [20, p.119] when $m+q \geq 6$.

Next we need a "completion" theorem for bounded embeddings (compare [6, §16]. Suppose that $Z$ is an open topological manifold, equipped with a reference map to an open cone $O(K)$, so that $p: Z \rightarrow O(K)$ is a bounded $C W$ complex [5, Def. 1.5]. The $K$-completion of $Z$ is the disjoint union $\hat{Z}_{K}=Z \amalg K$ with a basis for the topology given by (i) open sets in $Z$, and (ii) sets of the form $p^{-1}(U \times(t, \infty)) \amalg U$, where $t \geq 0$ and $U \subset K$ is open.

We will be interested in comparing the local properties of this completion, when $Z$ is replaced by a manifold $Z^{\prime}$, bounded homotopy equivalent to $Z$ over $O(K)$. The main ingredient is the following

Lemma 2.2. Let $X \subset S^{n}$ be a finite simplicial subcomplex, where $n \geq 5$. Then the bounded structure set $\mathcal{S}\left(\begin{array}{c}S^{n}-X \\ \downarrow \\ O(X)\end{array}\right)$ has only one element.

Proof. Let $D X$ denote the Spanier-Whitehead dual of $X$ in $S^{n}$. We use the bounded surgery exact sequence [6]

$$
\cdots \rightarrow\left[\Sigma\left(D X_{+}\right), F / T o p\right] \rightarrow L_{n+1}\left(\mathcal{C}_{O(X)}(\mathbb{Z})\right) \rightarrow \mathcal{S}\left(\begin{array}{c}
S^{n}-X \\
\downarrow \\
O(X)
\end{array}\right) \rightarrow[D X, F / T o p] \rightarrow \ldots
$$

If $X=*$ or $X=S^{k}$ for some $k<n, k \neq n-2$ then crossing with $\mathbb{R}$ induces an isomorphism on the simply-connected $L$-groups and at the normal space level. For $k=n-2$ it is enough (for our later applications) to assume that the embedding of $X=S^{n-2}$ in $S^{n}$ is unknotted. Then the effective fundamental group is $\pi_{1}\left(S^{n}-X\right)=$ $\mathbb{Z}$ so the term behind the structure set is

$$
\pi_{2}(F / T \text { op }) \rightarrow L_{n+1}\left(\mathcal{C}_{\mathbb{R}^{n-1}}(\mathbb{Z}[\mathbb{Z}])\right) \cong L_{2}(\mathbb{Z}[\mathbb{Z}])
$$

But since $L_{2}(\mathbb{Z}[\mathbb{Z}])=L_{2}(\mathbb{Z})$ this assembly map is an isomorphism and we are done. For the general case, we write $X=Y \cup D^{k}$ and assume that the result is true for $Y$. We compare the assembly maps for $Y$ to $X$, where the third term involves "germs away from $Y$ ", and reduces to the case of $S^{k}$ handled above [9,3.11]. Indeed, on structure sets we have the bijections:

$$
\mathcal{S}\left(\begin{array}{c}
S^{n}-X \\
\downarrow \\
O(X)
\end{array}\right)_{>O(Y)} \simeq \mathcal{S}\left(\begin{array}{c}
S^{n}-S^{k} \\
\downarrow \\
O\left(S^{k}\right)
\end{array}\right)_{>O(*)} \simeq \mathcal{S}\left(\begin{array}{c}
S^{n}-S^{k} \\
\downarrow \\
O\left(S^{k}\right)
\end{array}\right)
$$


where the last step follows by an Eilenberg swindle, showing that $L_{i}\left(\mathcal{C}_{O(*)}(\mathbb{Z})\right)=0$ for all $i \geq 0$. We now consider the commutative diagram



The $Y$-assembly map is an isomorphism by induction, and the assembly map "away from $Y$ " is an isomorphism by the preliminary case above. A similar result holds for the map $[\Sigma(D X), F / T o p] \rightarrow L_{n+1}\left(\mathcal{C}_{O(X)}(\mathbb{Z})\right)$ and so the structure set is trivial.

When $(Z, \partial Z)$ is a topological manifold with boundary, we can also consider a relative $(K, L)$ completion in which $p: Z \rightarrow O(K)$ is a bounded $C W$ complex and $\partial p: \partial Z \rightarrow O(L)$ is a bounded $C W$ complex with respect to a subcomplex $L \subset K$. If $(F, \partial F):\left(Z^{\prime}, \partial Z^{\prime}\right) \rightarrow(Z, \partial Z)$ is a bounded homotopy equivalence of pairs over

$$
(p, \partial p):(Z, \partial Z) \rightarrow(O(K), O(L))
$$

then $(F, \partial F)$ extends to a homotopy equivalence $(\bar{F}, \partial \bar{F}):\left(\hat{Z}_{K}^{\prime}, \partial \hat{Z}_{L}^{\prime}\right) \rightarrow\left(\hat{Z}_{K}, \partial \hat{Z}_{L}\right)$ of pairs by taking the identity on $K$ and $L$.

Definition 2.3. The completed map $\bar{F}: \hat{Z}_{K}^{\prime} \rightarrow \hat{Z}_{K}$ is homotopic to a local homeomorphism (relative to $L$ ) near $K$, extending the identity on $K$, if there exists a neighbourhood $U \subset \hat{Z}_{K}$ of $x \in K$ such that $F$ restricted to $F-1(U-U \cap K)$ is boundedly homotopic over $O(K)$ to a homeomorphism. When $x \in L$ we further require that $\partial F$ be a local homeomorphism near $L$, and that $\partial F$ be fixed under the bounded homotopy.

In our applications there is a free $G$-action on $Z, Z^{\prime}$, and a $G$-action on $K$ so that and $p: Z \rightarrow O(K)$ is $G$-equivariant. If $F$ is a bounded $G$-homotopy equivalence so that $Z$ is a finite free bounded $G-\mathrm{CW}$ complex then $\bar{F}$ is a $G$-homotopy equivalence extending the identity on the $G$-invariant subset $K$ of both domain and range.

Theorem 2.4. Let $F: Z^{\prime} \rightarrow Z$ be a bounded homotopy equivalence of (open) topological $(m+q)$-manifolds, bounded over the open cone $O(K)$, where $K$ is a finite complex of dimension $m$. Suppose that the $K$-completion $\hat{Z}_{K}$ is a topological $(m+q)$-manifold with $m+q \geq 5$. Then the $K$-completion $\hat{Z}_{K}^{\prime}$ is also a topological $(m+q)$-manifold. Moreover, $\bar{F}$ is homotopy equivalent to a local homeomorphism near $K$ extending the identity on $K$.

This result also has a relative version. 
Theorem 2.5. Let $F:\left(Z^{\prime}, \partial Z^{\prime}\right) \rightarrow(Z, \partial Z)$ be a bounded homotopy equivalence of topological $(m+q)$-manifolds with boundary, bounded over $(O(K), O(L))$ where $L \subset$ $K$ a finite subcomplex with $\operatorname{dim} L<m$. Suppose that the $(K, L)$-completion $\left(\hat{Z}_{K}, \partial \hat{Z}_{L}\right)$ is a topological $(m+q)-$ manifold with boundary and $m+q \geq 6$. If $\partial \bar{F}$ is a local homeomorphism near $L$ extending the identity on $L$, then $\bar{F}$ is homotopy equivalent to a local homeomorphism (relative to $L$ ) near $K$, extending the identity on $K$.

Proof. We will prove the first result and leave the relative version to the reader. Let $x \in K$ and choose an open disk $D^{m+q} \subset \hat{Z}_{K}$ around $x$. Let $L=\operatorname{cls}\left(D^{m+q} \cap K\right)$ and $X=L / \partial L$. Since

$$
\mathcal{S}\left(\begin{array}{c}
S^{m+q}-K \\
\downarrow \\
O(K)
\end{array}\right)_{>O(K-L)} \simeq \mathcal{S}\left(\begin{array}{c}
S^{m+q}-X \\
\downarrow \\
O(X)
\end{array}\right)_{>O(*)}
$$

the result follows from the computation of the local structure sets in Lemma 2.2.

\section{BOUNDED SURGERY}

In this section, the existence of a non-linear similarity $V_{1} \oplus W \sim_{t} V_{2} \oplus W$ will be related to the kernel of a bounded transfer map introduced in $[9, \S 3]$. For background on bounded surgery we refer to [6].

We begin with an observation from [10, 1.7]: if $V_{1} \oplus W \sim_{t} V_{2} \oplus W$, then our inductive assumptions imply that there is a $G$-homeomorphism

$$
h: V_{1} \oplus W \rightarrow V_{2} \oplus W
$$

such that

$$
h \mid \bigcup_{H \neq 1} W^{H}
$$

is the identity. One easy consequence (see [1]) is

Lemma 3.1. There exists a $G$-homotopy equivalence $S\left(V_{2}\right) \rightarrow S\left(V_{1}\right)$.

Proof. If we 1-point compactify $h$ we obtain a $G$-homeomorphism

$$
h^{+}: S\left(V_{1} \oplus W \oplus \mathbb{R}\right) \rightarrow S\left(V_{2} \oplus W \oplus \mathbb{R}\right) .
$$

After an isotopy, the image of the free $G$-sphere $S\left(V_{1}\right)$ may be assumed to lie in the complement $S\left(V_{2} \oplus W \oplus \mathbb{R}\right)-S(W \oplus \mathbb{R})$ of $S(W \oplus \mathbb{R})$ which is $G$-homotopy equivalent to $S\left(V_{2}\right)$.

Let $f: S\left(V_{2}\right) / G \rightarrow S\left(V_{1}\right) / G$ denote the induced homotopy equivalence of the quotient lens spaces. Since for $\operatorname{dim} S\left(V_{i}\right)=1$ it is clear that $G$-homotopy equivalence implies $V_{1} \cong V_{2}$, we may assume that $\operatorname{dim} V_{i} \geq 4$. Another consequence is

Lemma 3.2. There exists an isovariant $G$-h-cobordism between $f * 1: S\left(V_{2} \oplus W\right) \rightarrow$ $S\left(V_{1} \oplus W\right)$ and the identity on $S\left(V_{1} \oplus W\right)$, which is a product on all the singular strata $S\left(W^{H}\right)$ for $H \neq 1$. 
Proof. This uses a special case of $[4,1.1]$. After radial re-scaling, we may assume that

$$
h\left(D\left(V_{1} \oplus W\right)\right) \subset \operatorname{int} D\left(V_{2} \oplus W\right)
$$

and the region

$$
\bar{Z}=D\left(V_{2} \oplus W\right)-\operatorname{int} h\left(D\left(V_{1} \oplus W\right)\right)
$$

is then an isovariant $G$-h-cobordism from some isovariant $G$-homotopy equivalence $g: S\left(V_{2} \oplus W\right) \rightarrow S\left(V_{1} \oplus W\right)$ to the identity. Since $h$ was the identity on all singular strata, it is not hard to check that $g$ and $f * 1$ are isovariantly $G$-homotopy equivalent, so we may assume that $g=f * 1$. Moreover, the cobordism $\bar{Z}$ is a product $S\left(W^{H}\right) \times I$ along the $H$-fixed sets for all $H \neq 1$ and a bounded free $G$-h-cobordism on the complement.

Corollary 3.3. The kernel of the bounded transfer map

$$
\operatorname{trf}_{W}: \quad \mathcal{S}^{h}\left(S\left(V_{1}\right) / G\right) \rightarrow \mathcal{S}^{b, h}\left(\begin{array}{c}
S\left(V_{1}\right) \times{ }_{G} W \\
\downarrow \\
W / G
\end{array}\right)
$$

contains the element $[f] \in \mathcal{S}^{h}\left(S\left(V_{1}\right) / G\right)$.

Proof. If the whole sphere $S(W)$ is singular (i.e. contains no free orbits), then the vanishing of the bounded transfer $\operatorname{trf}_{W}([f])$ follows immediately from Lemma 3.2 by removing $S(W) \times I$ from domain and range of the $G$-h-cobordism. This implies for example that in the present argument we may assume $\operatorname{dim} W \geq 2$, and $\operatorname{so} \operatorname{dim}\left(V_{i} \oplus\right.$ $W) \geq 6$, since $\operatorname{dim} W=1$ implies that $W=\mathbb{R}$. Later we will see in Corollary 3.5 that $\operatorname{trf}_{\mathbb{R}}([f]) \neq 0$ and so non-linear similarities do not occur for $\operatorname{dim}\left(V_{i} \oplus W\right) \leq 5$.

In general the problem is that the given $G$-h-cobordism may not restrict to a $G$-hcobordism of $S(W) \times I$. Let

$$
\bar{F}:\left(\bar{Z}, \partial_{-} \bar{Z}, \partial_{+} \bar{Z}\right) \rightarrow\left(S\left(V_{1} \oplus W\right) \times I, S\left(V_{1} \oplus W\right) \times 0, S\left(V_{1} \oplus W\right) \times 1\right)
$$

be the $G$-homotopy equivalence of triads given by (3.2) such that $\left.\bar{F}\right|_{\partial_{-} \bar{Z}}=i d$ and

$$
\left.\bar{F}\right|_{\partial_{+} Z}=f * 1: S\left(V_{2} \oplus W\right) \rightarrow S\left(V_{1} \oplus W\right) .
$$

In addition, we can assume that for each $1 \neq H \subset G$

$$
\left.\bar{F}\right|_{\bar{F}^{-1}\left(S(W)^{H} \times I\right)}
$$

is a homeomorphism whose restriction to $S(W)^{H} \times \partial I$ is the identity. Let

$$
X=\bigcup_{1 \neq H \subset G} S(W)^{H}
$$

denote the singular set of $S\left(V_{1} \oplus W\right)$, and

$$
U=\bar{Z}-\bar{F}^{-1}(X \times I)
$$


denote the complementary free stratum. The restriction of $\bar{F}$ to this open submanifold gives

$F:\left(U, \partial_{-} U, \partial_{+} U\right) \rightarrow\left(S\left(V_{1} \oplus W\right) \times I-X \times I, S\left(V_{1} \oplus W\right) \times 0-X \times 0, S\left(V_{1} \oplus W\right) \times 1-X \times 1\right)$

which is a free bounded $G$-h-cobordism, bounded over the open cone $O(X \times I)$.

Since $\operatorname{dim} V_{i} \geq 4$ we can regard the bounded $G$-homotopy equivalence $F$ as a bounded codim $\geq 3$ Poincaré embedding of $(S(W)-X) \times I$ in $U$, relative to the given embedding on $\partial_{ \pm} U$. By Theorem 2.1, there exists a free topological $G$-embedding inducing the given Poincaré embedding, and extending the embeddings already fixed on $\partial_{ \pm} U$.

By homotopy extension, we can assume that $F$ restricted to this embedding of $(S(W)-X) \times I \subset U$ is a bounded $G$-h-cobordism, relative to the identity on $\partial_{ \pm} U$, and $F$ is a bounded $G$-homotopy equivalence over $O(X \times I)$. Now we apply the "completion" construction of Section 2 to adjoin $X \times I$ to both domain and range. By Theorem 2.5 the result is a (new) compact $G$-h-cobordism

$$
\bar{F}^{\prime}:\left(\bar{Z}^{\prime}, \partial_{-} \bar{Z}^{\prime}, \partial_{+} \bar{Z}^{\prime}\right) \rightarrow\left(S\left(V_{1} \oplus W\right) \times I, S\left(V_{1} \oplus W\right) \times 0, S\left(V_{1} \oplus W\right) \times 1\right)
$$

between $f * 1: S\left(V_{2} \oplus W\right) \rightarrow S\left(V_{1} \oplus W\right)$ and the identity on $S\left(V_{1} \oplus W\right)$, with the additional property that the restriction of $\bar{F}^{\prime}$ to $\bar{F}^{\prime-1}(S(W) \times I)$ gives a $G$-h-cobordism with range

$$
(S(W) \times I, S(W) \times 0, S(W) \times 1)
$$

Now the complement

$$
Z^{\prime}=\bar{Z}^{\prime}-\bar{F}^{\prime-1}(S(W) \times I)
$$

is a free bounded $G$-h-cobordism between $f \times 1: S\left(V_{2}\right) \times W \rightarrow S\left(V_{1}\right) \times W$ and the identity on $S\left(V_{1}\right) \times W$, bounded with respect to the second factor projection to $W=O(S(W))$. By the definition of the bounded structure set, this means that $\operatorname{trf}_{W}([f])=0$ as required.

By comparing the ordinary and bounded surgery exact sequences $[9,3.16]$, and noting that the bounded transfer induces the identity on the normal invariant term, we can assume that $f$ has normal invariant zero. Therefore, under the natural map

$$
L_{n}^{h}(\mathbb{Z} G) \rightarrow \mathcal{S}^{h}\left(S\left(V_{1}\right) / G\right),
$$

where $n=\operatorname{dim} V_{1}$, the element $[f]$ is the image of $\sigma(f) \in L_{n}^{h}(\mathbb{Z} G)$, obtained as the surgery obstruction (relative to the boundary) of a normal cobordism from $f$ to the identity. The element $\sigma(f)$ is well-defined in $L_{n}^{h}(\mathbb{Z} G)=\operatorname{coker}\left(L_{n}^{h}(\mathbb{Z}) \rightarrow L_{n}^{h}(\mathbb{Z} G)\right)$.

Lemma 3.4. For any choice of normal cobordism between $f$ and the identity, the surgery obstruction $\sigma(f)$ is a nonzero element of infinite order in $\tilde{L}_{n}^{h}(\mathbb{Z} G)$. 
Proof. Since $G$ is cyclic and $V_{1}$ is a free representation, the quotient $X=S\left(V_{1}\right) / G$ is a classical lens space of odd dimension $n-1$. An element in the surgery obstruction group $\tilde{L}_{2 k}^{h}(\mathbb{Z} G)$ is determined by its discriminant $D$ and multi-signature $\sigma$ : the odd order case is [20,13A.5] and the even order case is similar, based on the fact that $\tilde{L}_{2 k}^{s}(\mathbb{Z} G)$ is torsion-free. If $X, X^{\prime}$ differ by a normal cobordism, then [20, 14E.8] gives the relations $\Delta\left(X^{\prime}\right)=D \Delta(X)$ and $\rho\left(X^{\prime}\right)=\sigma+\rho(X)$, where $\Delta(X)$ is the Reidemeister torsion $\rho(X)$ is the $\rho$-invariant $[20,14 \mathrm{E} .7]$. Both $\Delta$ and $\rho$ are multiplicative on joins.

If $\sigma(f) \in \tilde{L}_{n}^{h}(\mathbb{Z} G)$ were a torsion element, then the relations above would show that a suitable join of copies of $X=S\left(V_{1}\right) / G$ is $h$-cobordant to the corresponding join of copies of $X^{\prime}=S\left(V_{2}\right) / G$. But this would imply that $V_{1} \oplus \cdots \oplus V_{1} \sim_{t} V_{2} \oplus \cdots \oplus V_{2}$ and since these are free representations, that $V_{1} \cong V_{2}$.

Corollary 3.5. Under the natural map $L_{n}^{h}(\mathbb{Z} G) \rightarrow L_{n}^{p}(\mathbb{Z} G)$, the image of $\sigma(f)$ is nonzero.

Proof. The kernel of the map $L_{n}^{h}(\mathbb{Z} G) \rightarrow L_{n}^{p}(\mathbb{Z} G)$ is the image of $H^{n}\left(\mathbb{Z} / 2, \tilde{K}_{0}(\mathbb{Z} G)\right)$ which is a torsion group.

\section{THE TRANSFER MAP}

We will now study the transfer map $\operatorname{trf}_{W}$ in (3.3). Since localizing or completing at $p \nmid 2|G|$ gives an injection on the free part (see $[20, \S 13 \mathrm{~A}])$ :

$$
L_{n}^{h}(\mathbb{Z} G) \rightarrow L_{n}^{h}(\mathbb{Z} G) \otimes \mathbb{Z}_{(p)},
$$

and we intend to show that $\operatorname{trf}_{W}(\sigma(f)) \neq 0$ is a $p$-local injection for all $G$-representations $W$, we will lose no information about elements of infinite order by $p$-localizing all our $L$-groups. This will be assumed from now on, without changing notation.

Following $[7, \S 6],[14, \S 11 \mathrm{~b}]$, (see also $[9, \S 4]$ for previous applications in bounded topology) we denote the top component of our bounded surgery obstruction group by

$$
L_{n}^{h}\left(\mathcal{C}_{W, G}(\mathbb{Z})\right)(m)
$$

where $m=|G|$. The top component of a $p$-local Mackey functor $(p \nmid m)$ is the intersection of the kernels of all the restriction maps to proper subgroups of $G$. It turns out to be a natural direct summand of the $L$-group, associated to an idempotent in the $p$-local Burnside ring. Moreover the top component has the property that the images of maps induced on $L$-theory by the inclusion of proper subgroups, project trivially into the top component. In particular, after passing to the top component the indeterminacy in $\sigma(f)$ is zero. Then Theorem A is implied by

Theorem 4.1. For any $G$-representation $W$, with $W^{G}=W^{H}$ when $[G: H]=2$, and any $p \nmid 2|G|$, the transfer

$$
\operatorname{trf}_{W}: L_{n}^{h}(\mathbb{Z} G)(m) \rightarrow L_{n+k}^{h}\left(\mathcal{C}_{W, G}(\mathbb{Z})\right)(m)
$$

is a $p$-local injection, where $k=\operatorname{dim} W$. 
To begin the proof, we will assume that $W=\oplus W_{i}$ is a direct sum of irreducible 2-dimensional quotient representations. Each $W_{i}$ has kernel $H_{i} \neq 1$ which is a proper subgroup of $G$.

If $W=W_{1} \oplus W_{2}$ there is another inclusion map,

$$
c\left(W_{1}, W\right)_{*}: L_{n}^{h}\left(\mathcal{C}_{W_{1}, G}(\mathbb{Z})\right) \rightarrow L_{n}^{h}\left(\mathcal{C}_{W_{1} \oplus W_{2}, G}(\mathbb{Z})\right)
$$

induced by the subspace inclusion $W_{1} \subset W$.

Lemma 4.2. If $\left(W_{2}\right)^{G}=\{0\}$ then the subspace inclusion $W_{1} \subset W_{1} \oplus W_{2}$ induces an isomorphism

$$
L_{n}^{h}\left(\mathcal{C}_{W_{1}, G}(\mathbb{Z})\right)(m) \rightarrow L_{n}^{h}\left(\mathcal{C}_{W_{1} \oplus W_{2}, G}(\mathbb{Z})\right)(m)
$$

on the top component for all $n$.

Proof. The subspace inclusion sits in the exact sequence given in [9, 3.12], and the result is a special case of $[9,4.5]$.

Corollary 4.3. If $W^{G}=\{0\}$ then the "cone point" inclusion induces an isomorphism

$$
c_{*}: L_{n}^{h}(\mathbb{Z} G)(m) \rightarrow L_{n}^{h}\left(\mathcal{C}_{W, G}(\mathbb{Z})\right)(m)
$$

on the top component for all $n$.

Proof. This is just the special case $W_{1}=\{0\}$ and $[9,3.10]$.

We can now reduce to the case where $W$ is irreducible.

Lemma 4.4. Suppose that $W$ satisfies the assumptions above, and $W_{1} \oplus W_{2}$ where $\operatorname{dim} W_{i}=2 l_{i}$. If $\operatorname{trf}_{W_{i}}$, for $i=1,2$ induces a monomorphism

$$
\operatorname{trf}_{W_{i}}: L_{n}^{h}(\mathbb{Z} G)(m) \rightarrow L_{n+2 l_{i}}^{h}\left(\mathcal{C}_{W_{i}, G}(\mathbb{Z})\right)(m)
$$

on the top component for any $n$, then so does $\operatorname{trf}_{W}$.

Proof. First note that

$$
\operatorname{trf}_{W_{1} \oplus W_{2}}: L_{n}^{h}(\mathbb{Z} G) \rightarrow L_{n+2 l_{1}+2 l_{2}}^{h}\left(\mathcal{C}_{W_{1} \oplus W_{2}, G}(\mathbb{Z})\right)
$$

is the composite of

$$
\operatorname{trf}_{W_{1}}: L_{n}^{h}(\mathbb{Z} G) \rightarrow L_{n+2 l_{1}}^{h}\left(\mathcal{C}_{W_{1}, G}(\mathbb{Z})\right)
$$

and

$$
\operatorname{trf}_{W_{2}}: L_{n+2 l_{1}}^{h}\left(\mathcal{C}_{W_{1}, G}(\mathbb{Z})\right) \rightarrow L_{n+2 l_{1}+2 l_{2}}^{h}\left(\mathcal{C}_{W_{1} \oplus W_{2}, G}(\mathbb{Z})\right)
$$


The first map is a monomorphism on the top component by assumption, and the second can be studied by the commutative diagram

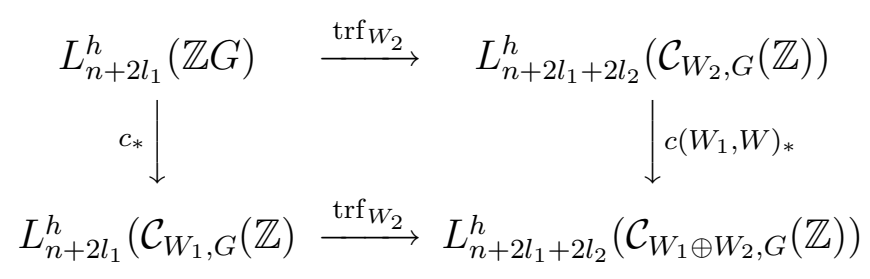

The horizontal maps are transfers $\operatorname{trf}_{W_{2}}$, and the vertical maps are induced by subspace inclusions. Since the subspace inclusions induce isomorphisms on the top component and the upper horizontal map is a monomorphism by assumption, we are done.

We now assume that $W$ is an irreducible 2-dimensional $G$-represention, with kernel $1 \neq H \neq G$. The $G$-equivariant projection $S\left(V_{1}\right) \times S(W) \rightarrow S\left(V_{1}\right)$ gives a circle bundle with fibre $S(W)=S^{1}$.

Lemma 4.5. The group $\Gamma_{H}=\pi_{1}\left(S\left(V_{1}\right) \times_{G} S(W)\right)$ is isomorphic to $\mathbb{Z} \times H$.

Proof. By pulling-back the circle bundle to the covering $S\left(V_{1}\right) / H \rightarrow S\left(V_{1}\right) / G$ we obtain a commutative diagram

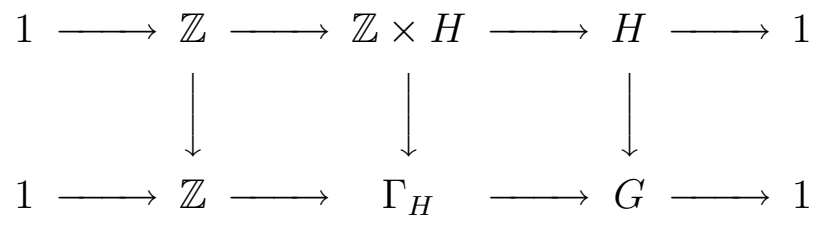

where the upper row is split exact. The result follows by the classification of extensions since $\operatorname{Ext}^{1}(G, Z)=H^{2}(G, \mathbb{Z}) \cong \mathbb{Z} / m$ and the distinct elements are given by the extensions

$$
1 \rightarrow \mathbb{Z} \stackrel{(k, 1)}{\longrightarrow} \mathbb{Z} \times \mathbb{Z} / d \stackrel{\left(\begin{array}{c}
1 \\
-k
\end{array}\right)}{\longrightarrow} \mathbb{Z} / m \rightarrow 1
$$

where $m=d k$. This extension is the unique one which splits when restricted to $\mathbb{Z} / d \subset \mathbb{Z} / m$.

In order to compute $\operatorname{trf}_{W}$ we will use the following diagram

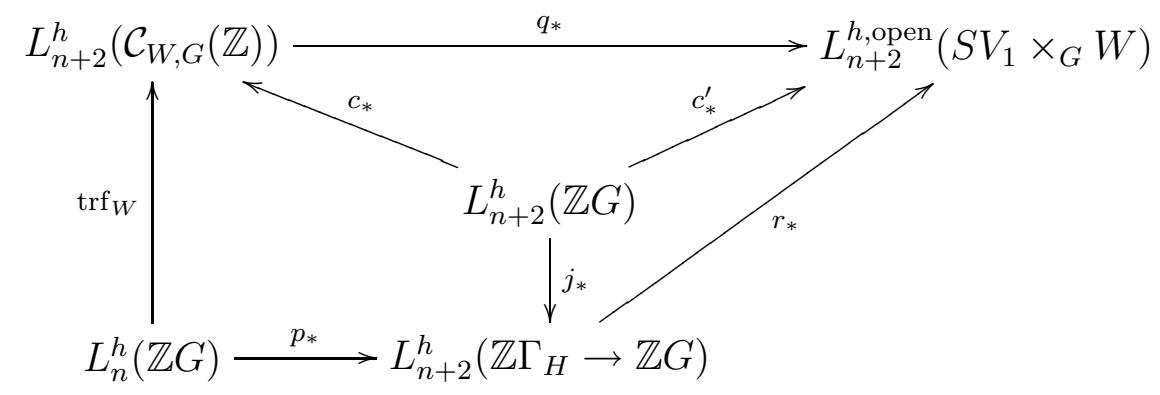


To construct this diagram we combine maps from three other surgery sequences with those for our bounded surgery groups.

(4.8): The first is just the exact sequence of the pair $\Gamma_{H} \rightarrow G$, corresponding geometrically to the inclusion of the circle bundle defined above into the total space of its disk bundle $S\left(V_{1}\right) \times_{G} D(W)$ :

$$
\cdots \rightarrow L_{n+2}^{h}\left(\mathbb{Z} \Gamma_{H}\right) \rightarrow L_{n+2}^{h}(\mathbb{Z} G) \rightarrow L_{n+2}^{h}\left(\mathbb{Z} \Gamma_{H} \rightarrow \mathbb{Z} G\right) \rightarrow L_{n+1}^{h}\left(\mathbb{Z} \Gamma_{H}\right) \rightarrow \ldots
$$

Let

$$
j_{*}: L_{n+2}^{h}(\mathbb{Z} G) \rightarrow L_{n+2}^{h}\left(\mathbb{Z} \Gamma_{H} \rightarrow \mathbb{Z} G\right)
$$

denote the relativization map.

(4.9): The second sequence arises from the theory of codimension two embeddings in $[20,11.6]$ :

$$
\ldots L S_{n}(\Phi) \rightarrow L_{n}^{h}(\mathbb{Z} G) \stackrel{p_{*}}{\longrightarrow} L_{n+2}^{h}\left(\mathbb{Z} \Gamma_{H} \rightarrow \mathbb{Z} G\right) \rightarrow L S_{n-1}(\Phi) \rightarrow \ldots
$$

where the obstruction groups were identified algebraically in $[16,7.8 .12]$ as the $L$ groups

$$
L S_{n}(\Phi) \cong L_{n}^{h}(\mathbb{Z}[\mathbb{Z} \times H], \beta, u)
$$

with respect to a "twisted" anti-structure $(\beta, u)$ for the ring $\mathbb{Z}[\mathbb{Z} \times H]$. In our situation, $\beta(\gamma)=\gamma^{-1}$ for all $\gamma \in \Gamma_{H}$ and $u=i_{*}(1)$ where $i_{*}: \mathbb{Z} \rightarrow \Gamma_{H}$ is induced by the inclusion $i: S(W) \rightarrow S\left(V_{1}\right) \times_{G} S(W)$ of the fibre. More explicitly, choose generators $a \in G$, $b=a^{k} \in H$ and $t \in \Gamma_{H}$ generating the infinite cyclic factor of $\Gamma_{H}=\mathbb{Z} \times H$ in the exact sequence (4.6). Then $u=t^{k} b \in \Gamma_{H}$.

Lemma 4.10. If $H \subset G$ is a non-trivial subgroup with $[G: H]>2$, then the image of $L S_{n}(\Phi) \rightarrow L_{n}^{h}(\mathbb{Z} G)$ is zero on the top component.

Proof. After applying the isomorphism $L S_{n}(\Phi) \cong L_{n}^{h}\left(\mathbb{Z}[\mathbb{Z} \times H], \beta, t^{k} b\right)$, we must compute the map

$$
L_{n}^{h}\left(\mathbb{Z}[\mathbb{Z} \times H], \beta, t^{k} b\right)(m) \rightarrow L_{n}^{h}(\mathbb{Z} G)(m)
$$

where the components for $\mathbb{Z} \Gamma_{H}$ are given by the preimages of proper subgroups of $G$ under the projection $\Gamma_{H} \rightarrow G$. There are two cases, depending on whether $k=[G: H]$ is even or odd. Note that we only need to discuss the case $n$ even, since the $p$-localization of the odd $L$-groups of $\mathbb{Z} G$ is zero.

First suppose that $k=2 l$ is even. Then by "scaling" the anti-structure we can assume that the unit $u=b$ and by $[17, \S 16]$ there is a natural direct sum splitting

$$
L_{n}^{h}(\mathbb{Z}[\mathbb{Z} \times H], \beta, b) \cong L_{n}^{h}(\mathbb{Z} H, \beta, b) \oplus L_{n-1}^{p}(\mathbb{Z} H, \beta, b) .
$$


Since $n$ is even, it is enough to compute the map induced by the inclusion

$$
L_{n}^{h}(\mathbb{Z} H, \beta, b) \rightarrow L_{n}^{h}(\mathbb{Z} G, \beta, b) \cong L_{n}^{h}(\mathbb{Z} G) .
$$

However, since $[G: H]=k>2$ there is a subgroup $H^{\prime}=\langle c\rangle$ with $b=c^{2}, H \subset H^{\prime} \subset G$ and $\left[H^{\prime}: H\right]=2$. Therefore our induction map factors through $L_{n}^{h}\left(\mathbb{Z} H^{\prime}, \beta, c^{2}\right) \rightarrow$ $L_{n}^{h}\left(\mathbb{Z} G, \beta, c^{2}\right)$ which is scale equivalent to the ordinary induction map (whose image has zero projection into the top component).

Next suppose that $k$ is odd. Since $H$ is a proper subgroup of $G$, with quotient $G / H \cong \mathbb{Z} / k$, the pullback of $H \subset G$ in $\Gamma_{H}$ is

$$
\Gamma_{H}^{\prime}=\mathbb{Z} \times H \stackrel{k \times 1}{\longrightarrow} \mathbb{Z} \times H \cong \Gamma_{H}
$$

from the description in Lemma 4.5. By scaling, $\left(\mathbb{Z}[\mathbb{Z} \times H], \beta, t^{k} b\right) \sim(\mathbb{Z}[\mathbb{Z} \times H], \beta, t b)$ so the induced anti-structures are the same on both $\Gamma_{H}$ and this proper subgroup $\Gamma_{H}^{\prime}$. But since $k$ is prime to $p$, the Mackey double coset formula shows that the induced restriction map on $L$-groups is a $p$-local injection. But since the $L$-groups are isomorphic and finitely generated by 4.9 , and the inclusion map integrally has at most 2-primary torsion in its cokernel, we conclude that

$$
L S_{n}(\Phi)(m) \cong L_{n}^{h}\left(\mathbb{Z}[\mathbb{Z} \times H], \beta, t^{k} b\right)(m)=0
$$

and $p_{*}$ is an isomorphism on the top component (compare [20, p.252] for a direct calculation in a special case).

(4.11): The third sequence comes from the "proper" surgery theory of MaumaryTaylor, resulting in surgery obstruction groups $L_{n}^{h \text {,open }}(K)$ where $K$ is a locally finite $C W$ complex. We will apply this to $K=S\left(V_{1}\right) \times_{G} W$ and use the Maumary exact sequence $[15,7.1]$

$$
\Pi_{n}^{h}(K) \stackrel{1-s}{\longrightarrow} L_{n}^{h}(\mathbb{Z} G) \oplus \Pi_{n}^{h}(K) \rightarrow L_{n}^{h, \text { open }}(K) \rightarrow \Pi_{n}^{p}(K) \rightarrow L_{n}^{p}(\mathbb{Z} G) \oplus \Pi_{n-1}^{p}(K)
$$

The terms

$$
\Pi_{n}^{q}(K)=\prod_{i=1}^{\infty} L_{n}^{q}\left(\pi_{1}\left(K_{i}\right)\right), \quad q=h, p
$$

where $K_{1} \supset K_{2} \supset K_{3} \supset \ldots$ is a sequence of neighbourhoods of infinity in $K$ so that each $K_{i}$ is cocompact and $\cap_{i=1}^{\infty} K_{i}=\emptyset$. In our case $K_{i}$ can be taken to be the product $S\left(V_{1}\right) \times_{G}\{w \in W \mid\|w\| \geq i\}$ so the fundamental groups are all isomorphic to $\Gamma_{H}$.

There are several natural maps relating the groups just introduced. We will need the following ones. 
(4.12): The compact relative surgery groups map into the proper groups: a relative problem can be modeled on the disk, sphere bundle pair $S\left(V_{1}\right) \times_{G}(D(W), S(W))$ and we can complete to the model $S\left(V_{1}\right) \times_{G} W$ by adding a ray $[1, \infty)$ at each point of $S(W)$. This gives

$$
r_{*}: L_{n+2}^{h}\left(\mathbb{Z} \Gamma_{H} \rightarrow \mathbb{Z} G\right) \longrightarrow L_{n+2}^{h, \text { open }}\left(S\left(V_{1}\right) \times_{G} W\right) .
$$

(4.13): There is a "cone point" inclusion

$$
c_{*}^{\prime}: L_{n+2}^{h}(\mathbb{Z} G) \rightarrow L_{n+2}^{h, \text { open }}\left(S\left(V_{1}\right) \times_{G} W\right)
$$

induced by the map $S\left(V_{1}\right) / G \times\{0\} \subset S\left(V_{1}\right) \times{ }_{G} W$. This map appears already in the Maumary exact sequence above.

Lemma 4.14. The "cone point" inclusion in (4.13) equals the composite

$$
L_{n+2}^{h}(\mathbb{Z} G) \stackrel{j_{*}}{\longrightarrow} L_{n+2}^{h}\left(\mathbb{Z} \Gamma_{H} \rightarrow \mathbb{Z} G\right) \stackrel{r_{*}}{\rightarrow} L_{n+2}^{h, o p e n}\left(S\left(V_{1}\right) \times_{G} W\right) .
$$

(4.15): There is a "forget some control" map

$$
q_{*}: L_{n+2}^{h}\left(\mathcal{C}_{W, G}(\mathbb{Z})\right) \rightarrow L_{n+2}^{h, \text { open }}\left(S\left(V_{1}\right) \times_{G} W\right)
$$

defined by regarding a bounded surgery problem as a proper surgery problem.

Lemma 4.16. The "cone point" inclusion in (4.13) equals the composite

$$
L_{n+2}^{h}(\mathbb{Z} G) \stackrel{c_{*}}{\longrightarrow} L_{n+2}^{h}\left(\mathcal{C}_{W, G}(\mathbb{Z})\right) \stackrel{q_{*}}{\longrightarrow} L_{n+2}^{h, \text { open }}\left(S\left(V_{1}\right) \times_{G} W\right) .
$$

where $c_{*}$ is the "cone point" inclusion from 4.3 .

Proposition 4.17. The outer square in diagram (4.7)

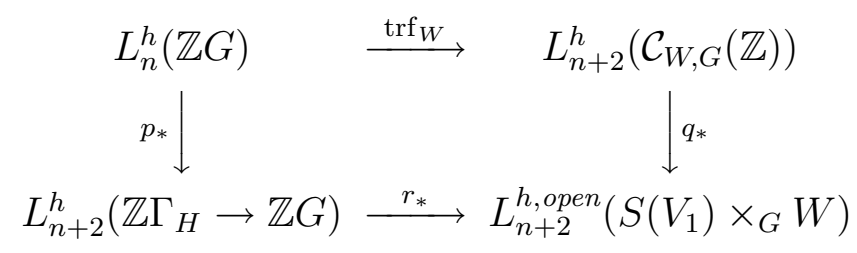

is commutative. The maps $r_{*}$ and $q_{*}$ induce an isomorphism on the top component, and the map $p_{*}$ induces a monomorphism on the top component. Hence $\operatorname{trf}_{W}$ is monic on the top component.

Proof. The commutativity of the diagram is easy to verify from the definitions of the maps given above. We will complete the proof below by checking the isomorphisms for $q_{*}, r_{*}$ and the monomorphism for $p_{*}$, implying the result for $\operatorname{trf}{ }_{W}$.

For $p_{*}$ we apply the top component idempotent to the exact sequence in (4.9). The idempotent comes from the family of proper subgroups of $G$, which gives by pull-back under the projection $\Gamma_{H} \rightarrow G$, a family of proper subgroups of $\Gamma_{H}$. Now the results of $[14, \S 11 \mathrm{~b}]$ produce a long exact sequence on the top components. But 
since $k$ is prime to $p$ and $[G: H] \neq 2$, Lemma 4.10 shows that $p_{*}$ is monic on the top component.

For $q_{*}$ we will use the Maumary exact sequence from 4.11 In our situation

$$
\Pi_{n}^{q}(K)=\prod_{i=1}^{\infty} L_{n}^{q}\left(\mathbb{Z} \Gamma_{H}\right), \quad q=h, p
$$

and a similar argument shows that the top component of this group is zero. Therefore the map $c_{*}$ in (4.13) is an isomorphism on the top component. Now by Corollary 4.3 and Lemma 4.16 the map $q_{*}$ is also an isomorphism on the top component.

For $r_{*}$ we use Lemma 4.14 to see that $r_{*}=c_{*} \circ j_{*}$ where $c_{*}$ is the "cone point" inclusion from (4.13), which we have already checked is an isomorphism on the top component. But $j_{*}$ sits in the exact sequence of 4.8 with the third term being $L_{n}^{h}\left(\mathbb{Z} \Gamma_{H}\right)$. Once again, this $L$-group is zero in the top component and so both $j_{*}$ and finally $r_{*}$ induce top component isomorphisms.

We now complete the proof of Theorem 4.1 by considering the bounded transfer map for representations which contain trivial subrepresentations (i.e. summands $\mathbb{R}^{k}$ on which $G$ acts trivially). In this case we again prove that trf induces a $p$-local monomorphism for $p \nmid 2|G|$.

Lemma 4.18. Suppose that $\operatorname{trf}_{W}: L_{n}^{h}(\mathbb{Z} G) \rightarrow L_{n+\operatorname{dim}(W)}^{h}\left(\mathcal{C}_{W, G}(\mathbb{Z})\right)$ is a p-local monomorphism on the top component, for all $n$. Then $\operatorname{trf}_{W \oplus \mathbb{R}}$ is also a p-local monomorphism on the top component, for all $n$.

Proof. The transfer $\operatorname{trf}_{W \oplus \mathbb{R}}$ can be identified with the natural "change of $K$-theory" map

$$
L_{n+\operatorname{dim}(W)}^{h}\left(\mathcal{C}_{W, G}(\mathbb{Z})\right) \rightarrow L_{n+\operatorname{dim}(W)}^{p}\left(\mathcal{C}_{W, G}(\mathbb{Z})\right)
$$

by means of the isomorphism

$$
L_{n+\operatorname{dim}(W)+1}^{h}\left(\mathcal{C}_{W \oplus \mathbb{R}, G}(\mathbb{Z})\right) \cong L_{n+\operatorname{dim}(W)}^{p}\left(\mathcal{C}_{W, G}(\mathbb{Z})\right)
$$

given in $[17, \S 15]$. But the kernel and cokernel of (4.19) are 2-torsion groups, since the change of $K$-theory map sits in a long exact sequence whose third term is $H^{*}\left(\mathbb{Z} / 2, \tilde{K}_{0}\left(\mathcal{C}_{W, G}(\mathbb{Z})\right)\right.$.

Corollary 4.20. For any $G$-representation $W$, with $W^{G}=W^{H}$ when $[G: H]=2$, the image $\operatorname{trf}_{W}(\sigma(f))$ under the bounded transfer is non-zero in the top component.

Proof. By Lemma 3.4 the element $\sigma(f)$ has infinite order. We can write any $G$ representation as a direct sum of irreducible 2-dimensional and trivial subrepresentations. By 4.2 and (4.17) the bounded transfer for the sum of the 2-dimensional factors is a $p$-local monomorphism on the top component., and the further transfer by a trivial representation $\mathbb{R}^{k}$ is a $p$-local monomorphism by (4). 
Remark 4.21. The change of $K$-theory map $L_{n}^{h}(\mathbb{Z} G) \rightarrow L_{n}^{p}(\mathbb{Z} G)$ is not an integral monomorphism in general for $G$ cyclic. If we had only odd index isotropy groups in our irreducible 2-dimensional representations $W$, then $\operatorname{tr} f_{W}$ would induce an isomorphism on the 2-local top components.

Proof of Corollary B. We may assume that $G$ is cyclic. If $G$ has odd order, then the result follows by induction from Theorem A. If $G$ has order $2 m$, where $m$ is odd then the odd order theorem determines half the characters of $V, V^{\prime}$ by restriction to the fixed set of the element of order 2 , and the other half by restriction to the subgroup of index 2. If $G$ has order $2^{l} m$, where $m$ odd and $l \geq 2$ and $V \sim_{t} V^{\prime}$ then by induction on $|G|$ we can assume that $\operatorname{Res}_{H} V \cong \operatorname{Res}_{H} V^{\prime}$ for all proper subgroups $H \subset G$. Since the elements of order $2^{l} \geq 4$ act trivially or freely in each irreducible subrepresentation of $V$, the fixed set $V^{\bar{H}}=V^{G}$ for the subgroup $H \subset G$ of index 2 . Now Theorem A gives the result.

\section{REFERENCES}

1. S. E. Cappell and J. L. Shaneson, Non-linear similarity, Ann. of Math. 113 (1981), 315-355.

2. _ The topological rationality of linear representations, Publ. Math. I. H. E. S. 56 (1983), 309-336.

3. S. E. Cappell, J. L. Shaneson, M. Steinberger, S. Weinberger, and J. West, The classification of non-linear similarities over $\mathbb{Z} / 2^{r}$, Bull. Amer. Math. Soc. 22 (1990), 51-57.

4. S. E. Cappell, J. L. Shaneson, M. Steinberger, and J. West, Non-linear similarity begins in dimension six, Amer. J. Math. 111 (1989), 717-752.

5. S. C. Ferry, I. Hambleton, and E. K. Pedersen, A survey of bounded surgery theory and applications, Algebraic Topology and Applications, Proceedings of MSRI conference 1990 (New York), Springer-Verlag, 1994, pp. 105-126.

6. S. C. Ferry and E. K. Pedersen, Epsilon surgery, Proceedings of Oberwolfach conference on the Novikov conjecture (S. C. Ferry, J. Rosenberg, and A. A. Ranicki, eds.), 1994, (To appear).

7. I. Hambleton and I. Madsen, Actions of finite groups on $R^{n+k}$ with fixed set $R^{k}$, Canad. J. Math. XXXVIII No 4 (1986), 781-860.

8. I. Hambleton and E. K. Pedersen, Topological equivalence of linear representations, To appear.

9. _ Bounded surgery and dihedral group actions on spheres, J. Amer. Math. Soc. 4 (1991), $105-126$.

10. W-C. Hsiang and W. Pardon, When are topologically equivalent representations linearly equivalent, Invent. Math. 68 (1982), 275-316.

11. N. Kuiper and J. W. Robbin, Topological classification of linear endomorphisms, Invent. Math. 19 (1973), 83-106.

12. I. Madsen and M. Rothenberg, On the classification of G-spheres I: equivariant transversality, Acta Math. 160 (1988), 65-104.

13. W. Mio, Nonlinearly equivalent representations of quaternionic 2-groups, Trans. Amer. Math. Soc. 315 (1989), 305-321.

14. R. Oliver, Whitehead Groups of Finite Groups, LMS Lecture Notes Series, vol. 132, Cambridge U. Press, Cambridge, 1988.

15. E. K. Pedersen and A. A. Ranicki, Projective surgery theory, Topology 19 (1980), 239-254.

16. A. A. Ranicki, Exact Sequences in the Algebraic Theory of Surgery, Math. Notes, vol. 26, Princeton University Press, Princeton N. J., 1981. 
17. L Lower K- and L-theory, Lond. Math. Soc. Lect. Notes, vol. 178, Cambridge Univ. Press, Cambridge, 1992.

18. G. de Rham, Reidemeister's torsion invariant and rotations of $S^{n}$, Diff. Analysis (Oxford), Oxford University Press, 1964, pp. 27-36.

19. C. Rourke and D. Sanderson, On topological neighborhoods, Comp. Math. 22 (1970), 387-424.

20. C. T. C. Wall, Surgery on Compact Manifolds, Academic Press, 1971.

Department of Mathematics \& Statistics, McMaster University, Hamilton, Ont., CANADA, L8S 4K1

Department of Mathematical Sciences SUny at Binghamton, Binghamton, Ny, 13901 